\title{
AN OVERVIEW ON MALARIA IN SUB-SAHARAN WITH SPECIAL REFERENCE TO TANZANIA
}

\author{
By \\ MAHFOUZ AHMAD AL-AGROUDI ${ }^{1}$, LAILA ABD EL-MAWLA MEGAHED ${ }^{1}$, \\ LAWRENCE TIA BANDA ${ }^{2}$ and TOSSON ALY MORSY ${ }^{3}$ \\ Military Medical Academy ${ }^{1}$, Tanzania People's Defence Forces ${ }^{2}$ and Department \\ of Parasitology, Faculty of Medicine, Ain Shams University, Cairo 11566 ${ }^{3,}$ Egypt \\ Abstract
}

Malaria is Anopheles-borne protozoan disease of worldwide distribution. Infection may result in a wide variety of symptoms, ranging from absent or very mild symptoms to severe disease and even death. Malaria can be categorized as uncomplicated or severe. It is a curable disease if diagnosed and treated promptly and correctly. All the clinical symptoms associated with malaria are caused by the asexual erythrocytic or blood stage parasites. When the parasite develops in the erythrocyte, numerous known and unknown waste substances such as hemozoin pigment and other toxic factors accumulate in the infected red blood cell. These are dumped into the bloodstream when the infected cells lyse and release invasive merozoites. The hemozoin and other toxic factors such as glucose phosphate isomerase (GPI) stimulate macrophages and other cells to produce cytokines and other soluble factors which act to produce fever and rigors and probably influence other severe pathophysiology associated with malaria.

After infective bite by Anopheles-vector, a period of time (incubation period) goes by before the first symptoms appear. Incubation period in most cases varies from 7 to 30 days. The shorter periods are most frequently with $P$. falciparum and the longer ones with $P$. malariae.

Antimalarial drugs taken for prophylaxis by travelers can delay the appearance of malaria symptoms by weeks or months, long after the traveler has left the malaria-endemic area. This can happen particularly with $P$. vivax and $P$. ovale, both of which can produce dormant liver stage parasites; the liver stages may reactivate and cause disease months after the infective mosquito bite. Besides, malaria-co-infection with HIV/AIDS and others is another serious issue.

Key words: Africa, Sub-Sahara, Malaria, Tanzania

\section{Introduction}

Malaria (= Quartan malaria; Falciparum malaria; Biduoterian fever; Black-water fever; Tertian malaria; Plasmodium) is an important cause of fever and serious illness in returned travelers. Among nearly 7000 returned travelers with fever seen at a GeoSentinel clinic between 1997 \&2006, for example, malaria was the most common specific etiologic diagnosis, found in $21 \%$ of cases. The relative risk of malaria is higher among returned travelers from the SubSaharan Africa than those from Asia or the Americas (Wilson et al, 2007). Sub-Saharan Africa is, geographically, the continent area of Africa that lies south of the Sahara (UN, 2010), consists of all African countries (54) that are fully or partially located south of the Sahara (Library of Congress, November 15, 2010).

Tanzania (Capital is Dodoma; Largest City
Is Dar es-Salaam); is the $13^{\text {th }}$ largest country in Africa and the $31^{\text {st }}$ largest in the world, ranked between the larger Egypt and smaller Nigeria. It borders Kenya and Uganda to the north; Rwanda, Burundi, and the Democratic Republic of the Congo to the west; and Zambia, Malawi and Mozambique to the south.

\section{Review, Discussion and Comment}

Approximately 1500 cases of imported malaria are reported annually to the CDC; this was likely an underestimate given underreporting in the United States (Mali et al, 2008). More than half of the reported cases are due to $P$. falciparum, which causes the most severe disease; patients with $P$. falciparum may progress to life-threatening illness within hours and it is associated with widespread drug resistance. Since 1997, there has been an average of six malaria deaths per year in the United States (Bruneel 
et al, 2003). Human Plasmodium malaria is caused by $P$. falciparum: P. vivax, $P$. ovale, $P$. malariae, and P. knowlesi (Cox-Singh et al, 2008). 1- P. falciparum causes the severest disease; patients with this form of malaria may progress to life-threatening illness within hours (Guerra et al, 2006). 2- P. vivax has the greatest geographic range and burden of disease. Worldwide, the estimated infections ranged between $130 \& 390$ million, with 2.6 billion individuals living at risk of infection (Kochar et al, 2005). Endemic vivax malaria occurs throughout most of the tropics, including Africa, Asia, the South Pacific, and Central and South America. It may occur at any latitude capable of supporting anopheline mosquitoes (even for brief periods) including temperate latitudes on the Korean Peninsula, China, Russia, and countries in southwestern Asia; as Iran, Afghanistan, and Tajikistan. Absence of Anopheles vector in tropical Micronesia and Polynesia spares these regions, and absence of Duffy factor on the surface of red blood cells among most Africans spares virtually all of west and central Africa of malaria due to $P$. vivax (Strickland, 2000). 3- P. ovale malaria was described in tropical western Africa and with rare frequency in Southeast Asia and Oceania. According to a survey performed in 1966, the only endemic areas for $P$. ovale malaria outside of Africa were the Philippine archipelago and the island of New Guinea. In a report of 15,806 blood film examinations at several sites in Indonesia between 1973 \& 1989, 34 infections due to $P$. ovale were identified; the frequency of $P$. ovale relative to $P$. falciparum and $P$. vivax was <1:1000 (Hay et al, 2004). 4- $P$. malariae occurs with relatively low prevalence in isolated pockets throughout the tropics (Mueller et al, 2007). 5- P. knowlesi is an emerging human pathogen initially recognized in 2004, although the first human case was described in 1965 (Chin et al, 1965). Malaria due to P. knowlesi was described in Malaysian Borneo; cases have also been reported in Thailand, Myanmar,
Singapore, and the Philippines (Putaporntip et al, 2009). However, P. knowlesi infection in macaques occurs in India, across Indochina, the Philippine archipelago, and the Indonesian archipelago to the island of Lombok, just east of Bali (Kantele and Jokiranta, 2011). P. knowlesi replicated every $24 \mathrm{~h}$ in human host and hence, causes quotidian malaria, causes a wide range of clinical pictures including fatal illness, and chloroquine is effective in treating uncomplicated malaria (Vadivelan and Dutta, 2014).

Humans living or traveling in all of these regions (e.g., within range of distribution of Anopheles vector), especially those living in proximity to macaques, may be considered at risk of infection by $P$. knowlesi (Luchavez et al, 2008). It appears to be a threat not only to the local population in Malaysia. Tourism resulted in increasing cases in Europe, America, and Oceania (Müller and Schlagenhauf, 2014) and to Germany from Thailand (Orth et al, 2013).

Clinical manifestations: Malaria should be suspected in the setting of any febrile illness after exposure to a region where malaria is endemic? (Wilson et al, 2007). Symptoms and signs of uncomplicated malaria may also include tachycardia, tachypnea, chills, malaise, fatigue, diaphoresis, headache, cough, anorexia, nausea, vomiting, abdominal pain, diarrhea, arthralgias and myalgias. The physical findings may include jaundice, splenomegaly and/or hepatomegaly. Moreover, manifestations of severe disease should prompt consideration of mixed infection with P. falciparum. These include hemodynamic instability, pulmonary edema, severe anemia, massive intravascular hemolysis, coagulopathy, hypoglycemia, metabolic acidosis, renal failure, hepatic dysfunction, altered mental status, focal neurological deficits and seizures. Febrile seizures are not a sign of severe malaria; a single seizure in the setting of a high temperature requires aggressive fever treatment with anti-pyretic to identify gnose a febrile seizure from seizures associated with the central nervous system 
complications of malaria (Ramharter et al, 2005).

Prevention efforts must include all human malaria; P. falciparum, $P$. vivax, $P$. ovale, $P$. malariae and $P$. knowlesi, which usually cause febrile illness but less commonly result in severe disease and are rarely fatal, although deaths have been reported in the setting of $P$. vivax and $P$. knowlesi infections (Cox-Singh et al, 2008). In general, most chemoprophylaxis regimens prevent primary malaria attacks, but may not prevent relapses that can occur with $P$. vivax and $P$. ovale. Most travelers who develop malaria do not adhere to an effective chemoprophylactic drug regimen, as many travelers fail to use personal protection measures for mosquito bite prevention (Svenson et al, 1995). Risk of malaria transmission depends on a variety of factors including the geographic region visited and type of traveler (Freedman, 2008). Besides the geographic region visited, malaria transmission risk depends upon the type of accommodation (e.g., open air, tented, air conditioned or screened), the season (rainy versus dry), the elevation, and the duration of exposure. The geographic risk assessment for malaria requires detailed review of the planned itinerary together with the most recent CDC guidelines and advisories. Listings of regions where malaria transmission occurs, the presence of the antimalarial drug resistance, and recommended chemoprophylaxis for specific destination are available in the CDC publication "Health Information for International Travel" (also known as the Yellow Book), which may be accessed online (CDC, 2008a).

Travelers: The important risk groups include travelers born in regions with endemic malaria that relocate outside the endemic area but subsequently return to visit friends and relatives (known as VFRs), pregnant women and military personnel.

VFR travelers are at greatest risk for malaria infection; this group includes individuals born in regions with endemic malaria who have emigrated outside these regions, as well as the subsequent family generation of children born outside endemic areas. VFRs pose unique challenges for malaria prevention since their acquired immunity afforded some degree of protection against malaria while they resided in the endemic area, although such immunity wanes outside endemic regions (Askling et al, 2005). Also, such individuals may have difficulty seeking or accessing preventive services, may receive incorrect information regarding appropriate prophylaxis measures, and may not appreciate the risk or severity of infection once their immunity has waned (Angell and Cetron, 2005).

Military personnel represent another important risk group; these individuals may have inadequate protection from mosquito bites given prolonged periods of nighttime exposure to biting Anopheles mosquitoes with accommodations that have inadequate screens or bed-nets (Ciminera and Brundage, 2007).

Counseling: Travelers to malarious areas should understand that their planned itinerary puts them at risk for malaria, a serious infection that can be fatal. Prevention measures include avoiding mosquito bites and adhering to the antimalarial chemoprophylaxis. Travelers must also understand that no chemoprophylaxis regimen guarantees complete protection and that fever during or after travel is a medical emergency requiring urgent medical attention. Other symptoms may include headache, myalgia, cough, nausea, abdominal pain, vomiting, and diarrhea.

Travelers should be counseled that their travel history is an important clue to bring to the attention of the health care provider in the first year following exposure. Because most chemoprophylactic agents do not eradicate the dormant hypnozoites of $P$. vivax and $P$. ovale can cause relapsing (with the exception of primaquine), infection with these species may present months following exposure in spite of full adherence to chemoprophylaxis. Among imported malaria cases in the United States in 2006, no cases of 
$P$. falciparum occurred more than six months following travel, although $18.1 \%$ of $P$. vivax cases occurred after this interval. However, in semi-immune individuals born in endemic areas, $P$. falciparum may present up to three years following travel (Schwartz et al, 2003). Travelers planning prolonged visits to endemic areas must continue prophylaxis during their stay. Local laboratories in developing regions may have high rates of false-positive malaria diagnoses; travelers who become ill should be advised to seek expert advice concerning malaria diagnosis and therapy (Keystone, 2004). In such cases the chemoprophylactic regimen should be continued together with treatment offered locally, unless significant drug-drug interactions (such as mefloquine with halofantrine). If initial evaluation showed negative blood films, thick and thin blood films should be repeated twice (12 to 24 hours apart).

Pregnant women are an important risk group, as malaria can be a life threatening infection for both mother and fetus. Risk of stillbirth, spontaneous abortion, and other adverse pregnancy outcomes is increased in the setting of malaria, and pregnant travelers should be advised to defer travel until after delivery whenever feasible.

Mosquito Bite Prevention: Travelers to malarious areas should receive instructions regarding methods to prevent bites from Anopheles mosquitoes; such measures also help reduce bites from sandflies, ticks and other mosquito species. These included avoiding outdoor exposure between dusk and dawn (at time of Anopheles feed) Wearing clothing that reduces the amount of exposed skin Wearing insect repellant Sleeping within bed nets treated with insecticide (permethrin) Staying in well-screened or airconditioned rooms (Fradin and Day, 2002). But, Apart from Anopheles vector, one must take into consideration that malaria could be transmitted by blood transfusion and needlestick injury (Saleh et al, 2017).

Insect repellents recommended by the $\mathrm{CDC}$ for reducing the risk of malaria include
DEET and picaridin. DEET (30 to 50\%) is generally protective for at least 4 hours, although lower percentage preparations provide a shorter duration of protection. When used appropriately, DEET is safe for infants and children over the age of 2 months. Picaridin is a synthetic repellant. This agent $(20 \%$ concentration) and DEET (35\% concentration) had comparable efficacy for protection against malaria vectors up to eight hours after application (Frances et al. 2004). The highest concentration of picaridin sold in the US is $15 \%$, and there is not sufficient data to support adequate protection against Anopheles at this concentration.

In addition to insect repellants applied to skin, fabric may be treated with permethrin or other residual insecticides. Permethrin is a synthetic compound that causes nervous system toxicity to insects with low toxicity for humans (Kimani et al, 2006). It is available in outdoor supply stores as an aerosol clothing spray (Permanente Repellent).

Clothing and bed netting treated with permethrin effectively repel mosquitoes for more than one week even with washing and field use. Standard nets dipped in permethrin proved effective for three washes whereas newer formulations can withstand 20 washes (Fradin, 1998).

Long lasting insecticide impregnated nets can remain effective as long as three years. Use of such nets is very effective for reducing the risk of malaria infection and travelers to the endemic areas with accommodations lacking screens or air conditioning (such as VFRs or hikers) should sleep under insecticide treated nets (Nevill et al, 1996).

Transmission other than by Anopheles spp.: Nosocomial or needle-stick injury: Cannon et al. (1972) reported transmission of $P$. falciparum via non-intact skin to Health care worker. Börsch et al. (1982) reported malaria transmission from patient to nurse via nonintact skin by needle-stick injury and Freedman (1987) in South Africa reported two nurses who acquired malaria by needlestick injury. Guerrero et al. (1983) and Nah- 
len et al. (1991) and (Mungai et al, 2001) in USA reported malaria transmission by blood transfusion. Herwaldt and Juranek (1993) in USA reported laboratory-acquired malaria, leishmaniasis, trypanosomiasis, and toxoplasmosis by needle-stick injury and $P$. cynomolgi transmission to laboratory researcher's normal skin by needle-stick injury. Piro et al. (2001) in Libya described two cases of malaria in a malaria-free zone in two patients, two weeks after a case of $P$. falciparum, acquired in Burkina Faso, had been admitted to the same ward. After reviewing the techniques used by nursing staff, concluded that transmission occurred via gloves contamination after manipulation of venous cannulas and drip lines of patient with Burkina Fasoacquired malaria that was not discarded before manipulating intravenous lines of the other two patients. Nosocomial transmission of unusual and potentially life-threatening infections should be taken into consideration in the settings where compliance with universal precautions is not rigorous (Saleh et al, 2016). Alweis et al. (2004) in USA reported a nurse who developed falciparum malaria after the needle-stick injury from a proved malignant malaria patient. Three days prior to her diagnosis, she cared for another patient, who subsequently developed falciparum malaria. That patient's parasite isolate genetically matched the nurse's isolate by two independent DNA fingerprinting techniques. They believed that the nurse acquired falciparum malaria via needle-stick subsequently transmitted malaria to another patient via a break in standard precautions.

Chemoprophylaxis: Individual itineraries should be reviewed together with the update guidelines and advisories to determine the appropriate approach to chemoprophylaxis. Listings of regions with risk for the malaria transmission, the presence of the antimalarial drug resistance, and recommended chemoprophylaxis were reported (CDC, 2008a).

Drug Mechanisms: There are three major categories of drugs for prevention of malaria, which target liver active tissue schizonts.
Atovaquone/ proguanil proved active against liver stages of $P$. falciparum.

Hypnozoiticide-Hypnozoites are a quiescent stage in liver that exists only in the setting of $P$. vivax and $P$. ovale infection. This liver stage does not cause clinical symptoms, but with reactivation and release into circulation, late-onset or relapsed disease can occur up to many months after initial infection.

Primaquine is active against the quiescent hypnozoites of $P$. vivax and $P$. ovale. This class of drugs targets the asexual blood stages of the parasite: chloroquine, mefloquine, doxycycline, atovaquone/proguanil and primaquine. As the first three agents act on blood parasites following release from the initial maturation phase in the liver, these drugs must be continued for four weeks following exposure to eradicate parasites released from the liver. Primaquine has schizonticidal activity against the tissue stages of all species and blood stages of the nonfalciparum species, but lacks blood schizonticidal activity against $P$. falciparum. Since atovaquone/ proguanil and primaquine act as both blood and tissue schizonticides, they interfere with development of actively replicating parasites in liver, so can be discontinued one week after the end of exposure.

Drug Resistance: Antimalarial selection should include the considerations of regions with malarial drug resistance (Wellems and Plowe, 2001). Individual itineraries should be reviewed together with the most recent guidelines and advisories.

Resistance of $P$. falciparum to chloroquine is widespread; regions with chloroquineresistant and chloroquine-sensitive malaria are summarized below: Chloroquine-resistant $P$. falciparum (CRPF) is widespread in endemic areas of Africa, Asia \& Oceania. Chloroquine-sensitive $P$. falciparum exists in the Caribbean, Central America, west and north of the Panama Canal, Mexico, and parts of North Africa, the Middle East, and China. $P$. falciparum strains resistant to chloroquine, mefloquine and sulfonamides are rare, but prevalent in the regions of Thai- 
land bordering Burma (Myanmar) and Cambodia (e.g., eastern provinces of Myanmar and western provinces of Cambodia, including Siem Reap), and in parts of China, Laos, and Vietnam. Chloroquine-resistant $P$. vivax is widespread in the Indonesian Papua and Papua New Guinea (Baird et al, 1996).

Regimens: Chemoprophylaxis agents vary with respect to cost, adverse effects, and dosing schedule. Reviewing these items during the travel visit is important for facilitating adherence. In 2006, 94\% of the US travelers with malaria took no chemoprophylaxis, took an ineffective drug, or took an appropriate drug incorrectly (Chen et al, 2008).

Antimalarial therapy should be started prior to travel, continued regularly during exposure, and for a period of time following departure from the endemic area. A prescription for the full supply of medication should be written and filled prior to departure; the sale of counterfeit and poor quality antimalarials is an increasing problem in Asia and Africa. Travelers should understand the importance of careful adherence to the chemoprophylaxis regimen, even though no chemoprophylaxis regimen guarantees complete protection.

1-Atovaquone-proguanil: Atovaquone acts synergistically with proguanil against chloroquine-sensitive and chloroquine-resistant $P$. falciparum, as well as the other malaria species that cause human malaria. Its efficacy is equivalent to that of mefloquine. Atovaquone-proguanil does not prevent hypnozoite formation by $P$. vivax or $P$. ovale; in areas with high of infection rates due to these species, presumptive anti-relapse therapy with primaquine may be necessary to prevent relapse for persons who had been to those areas for extended periods of time (Boggild et al, 2007). Atovaquone-proguanil is administered daily beginning two days prior to exposure, during exposure, and for one week following exposure. Drug is well tolerated, with excellent profiles of safety and efficacy (van Riemsdijk et al, 2002).

Side effects include gastrointestinal upset, insomnia, headache, rash and mouth ulcers.

Atovaquone-proguanil is contraindicated in patients with creatinine clearance $<30 \mathrm{~mL}$ per minute and it is not recommended for use in pregnant women due to insufficient safety data (McKeage and Scott, 2003).

2- Mefloquine: Mefloquine is effective for prevention of malaria due to chloroquinesensitive and chloroquine-resistant $\mathrm{P}$. falciparum, as well as the other malaria species that cause human malaria. In a study of 140,000 travelers to East Africa, the prophylactic efficacy of mefloquine was $91 \%$. Mefloquine is not effective for prevention of malaria due to mefloquine-resistant $P$. falciparum, which is found along the ThailandCambodian border region, and parts of China, Burma (Myanmar) and Vietnam. In addition, mefloquine does not prevent the development of residual hepatic hypnozoite forms of $P$. vivax or $P$. ovale malaria. For those with extended exposure to areas with high infection rates due to these species, presumptive anti-relapse therapy with primaquine may be necessary to prevent relapse. Mefloquine is administered weekly beginning at least two weeks prior to exposure, during exposure, and for four weeks following exposure. Some travelers experience adverse effects from mefloquine; most are mild, selflimited, and do not require drug discontinuation. Commonest adverse effects are gastrointestinal upset, lightheadedness, headache, difficulty concentrating, mood swings, strange dreams, and about $5 \%$ of them experience disabling neuropsychiatric adverse effects requiring discontinuation of the drug. These include anxiety, depression, nightmares, paranoid ideation and dizziness. Travelers about 1/10,000 experienced severe neuropsychiatric reactions; as seizures and psychosis. Adverse effects are the commonest among women and less among children. Most adverse ones requiring mefloquine discontinuation occur within first three doses. So, some favor start mefloquine four weeks prior to travel to determine drug tolerance.

Contraindications to mefloquine include 
known hypersensitivity to the drug, a history of seizures or major psychiatric disorder, and a recent history of depression or anxiety. Development of psychiatric symptoms (such as depression, anxiety, restlessness or confusion) while taking mefloquine should be viewed as a possible prelude to other events; in such circumstances it is advisable to stop drug immediately and switch to a different prophylaxis agent. Mefloquine was associated with sinus bradycardia and QT interval prolongation, and must be used with caution with cardiac conduction disorders patients.

For pregnant patients who cannot avoid travel to areas with chloroquine-resistant $P$. falciparum, limited data suggested that mefloquine might be safely administered during the second and third trimesters, and can probably also be administered during the first trimester (Nosten et al, 1994).

3- Doxycycline: Doxycycline has activity against chloroquine-sensitive and chloroquine-resistant $P$. falciparum, as well as the other malaria species that cause human malaria (Soto et al. 1998). Comparative trials have demonstrated equivalent efficacy of doxycycline with mefloquine (93-99\%). Doxycycline can provide some protection against infection with some rickettsial infections (e.g., scrub typhus) and Leptospira spp. However, doxycycline does not prevent the development of residual hepatic hypnozoite forms of $P$. vivax or P. ovale malaria. Thus, for those with extended exposure to areas with high rates of infection due to these species, presumptive anti-relapse therapy with primaquine may be necessary to prevent relapse. Doxycycline is administered daily beginning one to two days prior to exposure, daily during exposure, and daily for four weeks following exposure. Noncompliance with this daily regimen is an important reason for doxycycline prophylaxis failure (Wallace et al. 1996). Doxycycline is usually well tolerated, but was associated with gastrointestinal upset; less commonly, ultraviolet photosensitivity, Candida vaginitis, and rare cases of esophageal ulceration may also occur. The drug should be taken with fluids and food; and must not be given immediately before lying down. Sunscreen should be applied liberally for the duration of prophylaxis. It is advisable to offer women antifungal (fluconazole) self-treatment of C. vaginitis. Doxycycline is contraindicated in pregnant women and children less than 8 years old (Morris and Davis, 2000).

4- Chloroquine: Chloroquine may be used for prophylaxis for individuals traveling to malarious areas without chloroquine resistance. Chloroquine has activity against all Plasmodial species causing human malaria with the exception of chloroquine-resistant $P$. falciparum strains and uncommon strains of $P$. vivax in Oceania and Asia. Chloroquine did not prevent residual hepatic hypnozoite forms of $P$. vivax or $P$. ovale. So, for those with extended exposure to high rates of infection areas due to these species, must presumptive anti-relapse therapy with primaquine to prevent relapse. Formulations included chloroquine phosphate and hydroxychloroquine (CDC, 2008b). Chloroquine is administered once weekly starting one week prior to exposure, once weekly while in the malaria endemic area, and then once weekly for four weeks following exposure. Apart from its bitter taste, chloroquine is usually well tolerated. Minor side effects include gastrointestinal disturbances, dizziness, blurred vision, and headache; gastrointestinal problems may be alleviated by taking the drug with food. It has been associated with triggering flares of psoriasis and pruritis, although serious side effects are rare. Pruritus occurs in up to $25 \%$ of blacks due to the drug concentration in skin; this is not an allergic reaction. Retinal injury that can occur when high doses of chloroquine are used to treat rheumatoid arthritis, does not occur with weekly prevention dosa-ges used and safe for pregnancy (Salako, 1984).

5- Primaquine: Primaquine proved active against many stages of malaria parasite including hypnozoites, tissue schizonts, gametocytes, and asexual blood stages of $P$. vivax 
(Baird et al. 2003) in preventing relapses from hypnozoite forms of $P$. vivax and $P$. ovale; it also has activity against $P$. falciparum, including chloroquine-resistant species. The efficacy of primaquine against $P$. falciparum and $P$. vivax is 74 to $95 \%$ and 85 to $92 \%$, respectively. Primaquine may be used either as presumptive anti-relapse therapy (PART) or as primary prophylaxis. Primaquine primary prophylaxis is appropriate for travelers to regions where the principal endemic species is $P$. vivax (such as Mexico and Central America). Primaquine is administered daily beginning one to two days prior to exposure, once daily during exposure, and daily for seven days following exposure. Primaquine can cause hemolytic anemia in those with glucose-6-phosphate dehydrogenase (G6PD) deficiency. Thus, a G6PD level must be determined prior to administration of this drug and patients should receive primaquine only if G6PD deficiency was excluded. Primaquine may cause gastrointestinal upset minimized if taken with food. Primaquine is contraindicated in pregnancy and breastfeeding (Hill et al, 2006).

Relapse Prevention: Late-onset or relapsed disease due to reactivation of hypnozoites can occur up to many months after initial infection. Hypnozoites are a quiescent stage in the liver that exists only in the setting of $P$. vivax and $P$. ovale infection. According to CDC (2006) surveillance data, 220 travelers who took appropriate chemoprophylaxis were diagnosed with malaria; among the 80 cases of $P$. vivax or $P$. ovale in this group, $51 \%$ developed symptoms $>45$ days after arrival in the United States. Hepatic stages cause no fever symptoms and one does not need to have had a primary clinical episode of malaria in order to have a relapse.

Use of primaquine for presumptive antirelapse therapy (PART; the term terminal prophylaxis has also been used in the literature) is appropriate for travelers to regions where there is substantial risk of $P$. vivax or $P$. ovale transmission, even if $P$. falciparum is the predominant endemic species. This is especially appropriate for travelers with prolonged stays where $P$. vivax or $P$. ovale are present. In addition to administration of an agent with activity against blood stage parasites prior to, during, and following exposure (such as atovaquone-proguanil, mefloquine or doxycycline), primaquine is added for activity against liver hypnozoites which can cause disease after the completion of the primary chemoprophylaxis regimen (Galappaththy et al. 2007). After departure from an endemic area, primaquine should be administered for 14 days together with the remainder of the primary prophylaxis regimen. If the primary prophylaxis regimen has already been completed, a course of primaquine is still beneficial for prevention of relapse.

Pregnant Travelers: Malaria can be a life threatening infection for both mother and fetus. Risk of stillbirth, spontaneous abortion, and other adverse pregnancy outcomes is increased in the setting of malaria, and pregnant travelers should be advised to defer travel until after delivery whenever feasible (Lindsay et al, 2000). For pregnant women who cannot defer travel to regions where chloroquine-sensitive malaria is present, mosquito avoidance is a must in conjunction with chemoprophylaxis with chloroquine and Mefloquine is also acceptable (Smoak et al. 1997). For those who cannot defer travel to regions where chloroquine-resistant malaria is present, mosquito avoidance measures should be used with chemoprophylaxis using mefloquine (Okeyeh et al, 1996).

Limited data suggested that mefloquine may be safely administered during the second and third trimesters, and can probably also be administered during the first trimester. Safety data on atovaquone-proguanil in pregnancy are limited, so must be avoided. The doxycycline should not be administered during pregnancy because of potential adverse effects to the fetus including dysplasia and inhibition of bone growth and dental discoloration. Primaquine should not be administered during pregnancy given the potential possibility for fetal G6PD deficiency 
(Vanhauwere et al, 1998).

Drug interactions: The traveler's medication history should be reviewed in detail with consideration for potential drug-drug interactions. Some relatively common drugs with important interactions include: Warfarin: Atovaquone/ proguanil may diminish the metabolism of warfarin. Coagulation parameters should be monitored closely if these drugs are used together, and warfarin dosing may need to be reduced. Antiarrhythmic agents: these drugs should be used with caution in the setting of mefloquine administration, which has been associated with been associated with sinus bradycardia and QT interval prolongation. Protease inhibitors and non-nucleoside reverse transcriptase inhibitors: these agents may diminish metabolism of antimalarials and lead to potential interaction that may require the close monitoring, alteration of drug dosage or timing of administration (Khoo et al. 2005).

In light of limited data, doxycycline seems to have the least potential for drug-drug interactions with HIV drugs. Immunosuppressive medications: chloroquine may increase cyclosporine levels and both doxycycline and mefloquine may increase levels of cyclosporine and tacrolimus. Atovaquoneproguanil does not have known interactions with these medications (Kotton et al, 2005).

Suggested approach: Selection of chemoprophylaxis must be tailored to individual itineraries and circumstances. For patients traveling to several destinations with different types of malaria risk, it may be simplest to select a single agent that will be effective for the entire duration of exposure.

For travelers to destinations where malaria cases occur only sporadically and risk to travelers is very low, mosquito avoidance measures should be used; no chemoprophylaxis is needed. For travelers to destinations where chloroquine-resistant $P$. falciparum is present, mosquito avoidance measures must be used together with chemoprophylaxis.

The options include atovaquone/proguanil, mefloquine and doxycycline; all three agents proved highly efficacious for prevention of malaria. Comparative studies among travelers taking the mefloquine or atovaquone/ proguanil have demonstrated fewer side effects experienced among recipients of atovaquone/ pro-guanil, although this agent proved more costly (Camus et al, 2004). The short term travelers may prefer the shorter course of the atovaquone /proguanil, while long term tra-velers may prefer the convenience of weekly mefloquine. The doxycycline requires a prolonged course, must be taken daily, and causes sun sensitization, although it is the least expensive of these agents.

For travelers to destinations where chloroquine-sensitive $P$. falciparum malaria is present, mosquito avoidance is a must in conjunction with chemoprophylaxis.

Chloroquine may be used, although short term travelers may prefer shorter atovaquone /proguanil course. Mefloquine and doxycycline are also effective agents. For travelers to Mexico and Central America where $P$. vivax are dominant, mosquito avoidance is a must together with chemoprophylaxis. Primaquine may be used in absence of G6PD deficiency; chloroquine is also effective. Short term travelers prefer shorter atovaquone/proguanil course. Mefloquine and doxycycline are also effective agents.

For travelers to $P$. falciparum strains resistant to chloroquine, mefloquine, and sulfonamides are present as in malaria-endemic regions of Thailand bordering Burma (Myanmar) and Cambodia and western provinces of Cambodia, China, Laos, and Vietnam, mosquito avoidance is a must in conjunction with chemoprophylaxis. Options include atovaquone/ proguanil or doxycycline. The severity of hematologic disease is related to the ability of the malarial parasite to invade and grow in different red cell populations as well as the intrinsic growth rate of the parasite. 1- $P$. vivax and $P$. ovale have a strong preference to infect only young red cells (reticulocytes), thereby limiting parasitemia levels to approximately 1 to $2 \%$. Anemia 
due to hemolysis does occur and may be severe, but there is no peripheral sequestration of parasitized red cells. 2- P. malariae invades red cells of all ages, but parasite multiplication during each cycle is relatively low. Infection results in limited parasitemia ( $<1$ to $2 \%)$ and mild symptoms. 3- P. falciparum can invade red cells of all ages, including cells as early as orthochromatic erythroblasts (Tamez et al, 2009) multiplies 10-fold within each 24 hour cycle, and expresses clonally variant antigens on the surface of infected red cells, which are receptors for ligands on the surface of endothelial cells, red cells, and platelets. These variant antigens enable late blood-stage infected red cells to sequester in post-capillary venules. Parasitemia is often high, occasionally exceeding $50 \%$, and the potential for severe anemia, systemic disease, and death is considerable. 4- P. knowlesi was originally described as a malarial infection of macaque monkeys. Morphologically it resembles P. malariae and it appears that it causes significant and sometimes severe human infection, previously misdiagnosed as $P$. malariae, in Borneo, Malaysia, and in other areas of Southeast Asia and in travelers returning back (Cox-Singh et al, 2008).

Prevalence of anemia: Severe malarial anemia (SMA) is seen most frequently in areas of very high malarial transmission and most commonly in young children and pregnant women (Greenwood, 1997). The prevalence of anemia, defined as a hematocrit $<33 \%$, in malarial endemic areas of Africa varies between 31 and $91 \%$ in children, and between 60 and $80 \%$ in pregnant women (Schellenberg et al, 2003). In a typical study of children living a malarial endemic area in southern Cameroon, among those who were monitored for both asymptomatic and symptomatic malaria, the prevalence of anemia (hemoglobin $<11 \mathrm{~g} / \mathrm{dL}$ ) was the highest in a six-month-old age group (47\%), $42 \%$ in children $<3$ years of age and $21 \%$ in those $3-5$ years of age (Cornet et al, 1998). The placental malarial infection was the primary risk factor for the severe anemia in the sixmonth old infants.

In epidemiologic studies it was difficult to determine the number of cases of severe anemia attributable to malaria as the WHO definition of SMA is quite strict: a- Hemoglobin concentration $<5 \mathrm{~g} / \mathrm{dL}$ or hematocrit $<15 \%$, b- Parasitemia with $>10,000$ parasites/microL of blood, and c- Normocytic blood film (thus excluding thalassemia as well as iron, B12, and folate deficiencies). In patients, it may be difficult to attribute anemia to a single cause, although randomized placebo-controlled trials of malaria chemoprophylaxis and iron supplementation in infants have shown that malaria infection was the main etiologic factor for anemia. Also, intermittent anti-malarial treatment in pregnant women can substantially reduce the prevalence of severe maternal anemia (Schellenberg et al, 2001). The precise etiology of anemia is often complex in endemic areas since nutritional deficiencies, genetic traits, and intercurrent infection may all contribute to the anemia (Lamikanra et al, 2007). The etiology of SMA in endemic areas is likely to be multifactorial and variable in both time and place. This was illustrated in a case control study of severe anemia in hospitalized children in Malawi, severe anemia was associated with the following conditions (Calis et al, 2008): a-Malarial infection (odds ratio (OR) 2.3; 95\% CI 1.6-3.3), b- HIV infection (OR 2.0; 95\% CI 1.0-3.8), c- Hookworm (OR, 4.8; 95\% CI 2.0-12), d- Bacteremia (OR 5.3; 95\% CI 2.6-11), e- G6PD deficiency (OR 2.4; 95\% CI 1.3-4.4), and f- Deficiencies of vitamin A (OR 2.8; 95\% CI 1.35.8) and vitamin B12 (OR 2.2; 95\% CI 1.43.6). One must keep in mind the double and triple infection in Africa mainly Sub-Sahara.

Features of malarial anemia: The spectrum of the clinical presentation and severity of $\mathrm{P}$. falciparum infection is broad. In endemic areas many malarial infections present in semi-immune and immune children and adults as an uncomplicated febrile illness. Fever develops with the release of merozo- 
ites from ruptured, infected red cells. Anemia, thrombocytopenia, splenomegaly (occasionally massive), hepatomegaly, and jaundice can develop, and splenic rupture can occasionally occur (Bedu-Addo and Bates, 2002). However, SMA clinical setting varied and complex. Not only may acute infection present with anemia and/or cerebral malaria, respiratory distress and hypoglycemia, but chronic, repeated malarial infection may also lead to severe anemia. In either case, there may be a background of a low hemoglobin level due to the presence of other factors, as noted above, and malaria itself can predispose to bacteremia (Scott et al, 2011).

Severe malarial anemia: New malarial infections are often associated with a sudden drop in hemoglobin concentration associated with increased hemolysis and bone marrow suppression. Non-immune patients may exhibit a number of clinical syndromes including anemia, coma, respiratory distress, and hypoglycemia, and may have a high frequency of concurrent bacteremia (Berkley et al, 1999). Children may present with mild, moderate or even severe anemia with or without other syndromes of severe disease as malaise, fatigue, dyspnea, or respiratory distress as metabolic acidosis supervenes (English, 2000). The presentation of disease changes from severe anemia in children aged between one and three years in areas of high transmission to cerebral malaria in older children in areas of lower transmission. As transmission intensity declines, severe malaria is most frequently found in older age groups. There has been evidence of an increased incidence of anemia after malarial challenge in vaccine studies in non-human primates and also in field trials of blood stage vaccines (Ellis et al, 2011) The significance of these data remains uncertain, but there appears to be an increase in clearance of uninfected red blood cells during bloodstage infection in the vaccinated individuals.

Chronic anemia: Anemia is also present in those with chronic malarial infection. Many children might present with severe anemia and a blood smear negative for malaria parasites, but would respond to the antimalarial treatment (Roberts et al, 2005).

Hematologic features: The anemia of $P$. falciparum is typically normocytic and normochromic, with a notable absence of reticulocytes. Microcytosis and hypochromia may be present due to the very high frequency of thalassemia trait and/or iron deficiency in many, but not all, of the endemic areas (Prentice et al, 2012).

Black-water fever: A less common form of anemia in malaria is "Blackwater Fever" (BWF) characterized by intravascular hemolysis, the sudden appearance of hemoglobin in the urine, and renal failure, classically associated with irregular use of quinine. Disseminated intravascular coagulation and red cell fragmentation may accompany the presentation (Naqvi et al, 1996). An important clinical point is that BWF is sometimes difficult to relate to malarial infection, because parasitemia can be missed due to synchronous lysis of all infected red cells. Series of cases of BWF in Africa and South-East Asia have shown that sudden hemolysis is associated with malarial infection, glucose-6-phosphate dehydrogenase deficiency, and the use of quinine. BWF virtually disappeared after 1950, when chloroquine superseded quinine. However, in European expatriates living in Africa, BWF has been associated with use of the antimalarial agents' halofantrine, quinine, and mefloquine (Bruneel et al, 2001). The case series do not attempt to define the pathophysiological process(es) that underlie this syndrome. The oxidative stress might play an important role in some cases. Other mechanism(s) as drug-dependent antibodies were not well studied.

Anemia in $P$. vivax: Although most work describes the association of $\mathrm{P}$ falciparum malaria with anemia, infection with $P$ vivax can, on occasion, cause severe disease, including anemia and severe hemolysis. $P$. vivax malaria has been clearly associated with anemia during pregnancy, along with low birth weight of children of infected mo- 
thers (Rodríguez-Morales et al, 2006). Some studies in rural India suggest that severe disease is associated with $P$. vivax infection more frequently than with $P$. falciparum infection. Anemia is associated with increased red cell clearance, reticulocytopenia, and dyserythropoiesis (Panicha- kul et al, 2012).

Pathogenesis: Underlying causes of severe malarial anemia in humans may include one or more of the following mechanisms (Boele van Hensbroek et al, 2010): a- Extravascular clearance and/or intravascular destruction of infected RBCs, b- Clearance of uninfected RBC, c- Activation of monocy-te /macro-phage system, and d- Suppression of erythropoiesis along with dyserythropoiesis.

Host genetic factors: There is enormous variability among individuals in their response to malaria that may reflect host genetic factors. As an example, among West African ethnic groups, similar infection rates, morbidity, and antibody responses are found among the Mossi and Rimaibe in the Northeast of Ouagadougou in Burkina Faso (Modiano et al, 1996). In contrast, the Fulani, who live together and are exposed to the same hyperendemic transmission of $P$. falciparum, have less parasitemia, less morbidity, and a higher antibody response. The better outcome in the Fulani could not be explained by differences in the use of malaria protective measures, sociocultural or environmental factors, or genetic factors known to be associated with resistance to malaria.

Many genetic traits have been associated with protection from malaria. However, none of these associations has been consistently reported as specific for protection with one syndrome of malaria as opposed to others. For example, in a case-control study of malaria in West African children, a class II HLA haplotype (DRB1*1302-DQB1*0501), common in West Africa but much less frequent in other populations, was associated with protection from SMA due to P. falciparum, but not confirmed in other regions and the mechanism of protection has not been determined (Hill et al, 1992).
Malaria and Pregnancy outcome: Adverse maternal and perinatal outcomes associated with malaria during pregnancy include: 1Miscarriage, 2- Fetal growth restriction/ small for gestational age (SGA) infant, 3- Preterm birth ( $<37$ weeks of gestation), 4- Low birth weight $(\mathrm{LBW})(<2500 \mathrm{~g}$ at birth), 5Perinatal death, 6- Congenital infection, 7Maternal anemia, and 8-Maternal death

Miscarriage: An analysis of pregnancy outcome in over 17,000 pregnant women attending antenatal clinics at the ThaiBurmese border included 945 women with first trimester malaria, of whom 773 were treated with chloroquine, quinine, or artesunate .After adjustment for maternal age, previous miscarriages, smoking, and nonmalaria febrile illness, the odds of miscarriage in women with $P$. falciparum or vivax malaria were about three-fold higher than in women without malaria. The frequency of miscarriage in women without malaria, those with treated malaria, and all women with malaria (treated and untreated) was 19 , 27 , and $35 \%$, respectively. Miscarriage risk increased for symptomatic and asymptomatic malaria, but highest with symptomatic ones. Risk was similar for $P$ falciparum and $P$ vivax infection (McGready et al, 2012).

Preterm birth: The increased prevalence of preterm deliveries among women who become infected with $\mathrm{P}$. falciparum during pregnancy may be mediated by alterations in cytokine production. In particular, infected women have an increased concentration of tumor necrosis factor-alpha (TNF-alpha) in the intervillous circulation, and the concentration of TNF-alpha correlates with the density of $P$. falciparum-infected erythrocytes (Suguitan et al, 2003). Production of TNF-alpha plays a role in the pathogenesis of preterm birth and LBW. Individuals who carry the TNF 2 polymorphism in the promoter region of the TNF-alpha gene have heightened TNF-alpha production in response to infection, which increases their risk of preterm delivery, severe infection, and cerebral malaria (Aidoo et al, 2001). 
Low birth weight: In pregnancies complicated by malaria, both fetal growth restriction and preterm birth contribute to the LBW. Distinguishing between the two is challenging in resource-poor settings where ultrasound dating of pregnancy is usually not available and maternal recall of the last menstrual period is poor. In some settings, malaria has been estimated to be responsible for up to 70 percent of fetal growth restriction and up to $25 \%$ of LBW. Similar birth weight reductions and attributed fractions of malaria-associated LBW have been reported from areas of stable and unstable malaria transmission (Desai et al, 2007).

Impaired fetal growth is correlated most strongly with evidence of parasites in the placenta and the corresponding inflammatory infiltrate. It has been hypothesized that malarial infection of the placenta leads to placental thickening and fibrin deposition, thereby decreasing placental transport of oxygen and nutrients. Doppler studies have demonstrated impaired uteroplacental blood flow (Dorman et al, 2002). The mean birth weight of infants born to mothers with no evidence of malarial placental infection is higher than for those newborns from infected placentas (2763 vs. 2143 g). A significant decrease $(150 \mathrm{~g})$ in the average birth weight of infants of primigravidas with placental malaria has been reported, but this decrease was not significant $(52 \mathrm{~g})$ in offspring of the multigravidas (Salanti et al, 2004). This is related to the observation that primigravidas are more likely to have placental malaria and heavier parasite loads and are less likely to have partial immunity. Support for this theory was illustrated in a study that showed affected multigravidas had higher plasma levels of anti-VAR2CSA IgG, gave birth to markedly heavier babies, and had a significantly lower risk of delivering LBW children than affected primigravid women in whom anti-VAR2CSA IgG levels were low and placental sequestration was increased. The level of adhesion of placental parasites to chondroitin sulfate receptors also appears to be an important risk factor for LBW (Tuikue Ndam et al, 2004). The assumption of causality is confounded by the frequent concomitant presence of anemia in primigravidas with placental malaria (Brabin et $a l, 1990)$, which is independently associated with low birth weight. Indeed, when controlling for this confounding variable, the relationship between malaria and LBW becomes insignificant. However, since malaria is often the cause of the anemia, an association remains clinically compelling. A cohort study from Malawi including 2462 pregnant women found that an increasing number of $P$. falciparum infections during pregnancy increased the risks of both LBW and maternal anemia (Kalilani et al, 2010). Also, second trimester infection increased the risk of LBW more than third trimester infection.

Perinatal mortality: A systematic review of 117 studies published between 1948 and 2002 found that perinatal mortality (PNM) and fetal mortality were higher in malaria endemic countries than in nonendemic ones (PNM $61.1 / 1000$ vs. $25.8 / 1000 \&$ fetal mortality $40.1 / 1000$ vs. $20.0 / 1000$ ). In a subset of nine of these studies that correlated fetal demise with placental malaria, presence of the latter significantly increased the risk for stillbirth, regardless of the number of prior pregnancies; OR 2.19, 95\% CI 1.49-3.22 (van Geertruyden et al, 2004).

Congenital infection: All types of malaria can be transmitted congenitally, but congenital disease is most often associated with $P$. vivax and $P$. falciparum. Placental infection is a prerequisite for, but does not predict, congenital disease. Placental infection is more common than cord blood parasitemia, which is more common than parasites on the infant's peripheral smear. In Central African study of women who did not receive antimalarial agents during pregnancy, rates of placental infection, cord blood parasitemia \& infant's smear parasitemia were 74,6 , $\& 3.6 \%$, respectively (WHO, 2012). In the immune mothers, the risk of transplacental transmission of malaria appears to be small; 
0.1 to $1.5 \%$ of infants had cord blood positive for parasites \& clinical disease (Subramanian et al, 1992). The rate is somewhat higher in women with overt attacks during pregnancy ( 1 to $4 \%$ risk of congenital infection). The low incidence of fetal infection despite the known high incidence of placental infection is presumably secondary to passive immunization by transplacental acquisition of maternal antibody. Transplacental passage of malarial antigens capable of priming neonatal immune system has also been demonstrated (Metenou et al, 2007). In contrast, in semi-immune or nonimmune mothers, transplacental antibody transfer may be deficient, and congenital infection incidence was higher; 7 to $10 \%$. Also, maternal antibody levels were lower and congenital infection risk was higher in women who move from endemic areas to malariafree locales and no longer frequently exposed to infection (Soulard et al, 2011).

In endemic areas, it can be difficult to distinguish malaria acquired congenitally from that acquired as a newborn, particularly in infants of asymptomatic mothers. The onset of symptoms is usually at 2 to 8 weeks of age and includes poor feeding, fever, vomiting, diarrhea, and irritability. Anemia, thrombocytopenia, and hyperbilirubinemia are common. Splenomegaly is more common than hepatomegaly. There were five cases of congenital malaria reported in the United States since 2000. In one case report, the infected seven-month old infant presented with fever and anemia. Peripheral smears revealed malarial parasites with morphology consistent with $P$. vivax. Investigation by the public health department found that the mother had emigrated from Guatemala two years earlier and had a history of malaria with relapse while residing in her native country. The prenatal evaluation did not include any questions regarding a prior history of malaria, which may have contributed to the delay in diagnosis (CDC, 2005).

Maternal mortality: The WHO estimated that 10,000 maternal deaths each year are associated with malaria infection during pregnancy. Malaria is a leading cause of maternal mortality in regions of unstable endemism where there are periodic epidemics among nonimmune patients (Looareesuwan et al, 1985). In this series, $15.5 \%$ of the deaths were directly attributable to malaria, and $19.7 \%$ of the women who died were parasitemic with $P$. falciparum. Over onethird of the malaria-related deaths occurred in primigravid adolescents, primarily associated with severe anemia. Autopsies on 161 women with asymptomatic $P$. falciparum showed that $44(27.3 \%)$ had histological evidence of splenic malarial infection (Khan et $a l, 2014)$. A study performed at the main referral hospital in Gambia estimated that, during the malaria season, there was a $168 \%$ increase in the maternal mortality rate and a three-fold increase in the proportion of deaths due to anemia. It was estimated that malaria accounted for up to 93 maternal deaths per 100,000 live births (Anya, 2004). Younger maternal age has been associated with higher rates of anemia and poorer maternal and fetal outcome (Bouyou-Akotet et $a l, 2003)$. Adolescents have a significantly higher rate of anemia and SGA neonates than adults (anemia: 83 and 53\%, respectively; SGA: 50 versus $27 \%$, respectively). Adolescents in rural areas at earlier gestations are at higher risk of malarial infection and this infection is strongly associated with anemia (particularly in dry season). It suggests that control measures against malaria infection be targeted at younger rural early in pregnancy (Dicko et al, 2003).

Postpartum infection: The increased risk for both acquiring an infection and developing more severe disease persists for at least 60 to 70 days postpartum. Puerperal infection can also be caused by a new infection, rather than by parasites trapped in placenta and released into the maternal blood at delivery (Ramharter et al, 2005). The reason for high susceptibility to infection postpartum may be related to postpartum changes in the maternal immune system, the maternal- 
behavioral changes, or other undefined factors.

HIV/AIDS-Coinfection: The global burdens of malaria and human immunodeficiency virus (HIV) are high, together causing over 2.5 million deaths in 2009 (UN, 2012). Morrow et al. (1989) THE epidemic of human immunodeficiency virus type 1 (HIV-1) infection in areas of the tropics where Plasmodium falciparum is endemic has generated serious concern about potential interactions between the two infections. Ho and Webster (1989) reported that P. falciparum could occur more frequently or more severe in HIV-infected persons with profound CD4 lymphocyte depletion. From the standpoint of malarial disease, a seropositive HIV status increases the morbidity and mortality associated with the malaria infection. Mothers with HIV may be at higher risk for malaria acquisition, placental malaria, higher parasite densities, and more severe clinical disease. After delivery, women with HIV and malaria are at increased risk for anemia compared to HIV seronegative women with or without malaria. Dual infection with malaria and HIV also leads to an increased risk of adverse perinatal outcomes (Kublin et al, 2005). In many subSaharan African countries, including Tanzania, populations are at risk of HIV and malaria concurrently. However, action to tackle the two diseases is often carried out in parallel (WHO, 2010). The need for coordinated and integrated approaches is recognized as essential, and a focus has been placed on health systems as a whole in supporting programmes for different diseases (Janson, 2007). The negative clinical consequences of HIV-malaria co-infection are already well established, including a higher risk of malaria and developing more severe clinical symptoms amongst HIV-positive people, as reviewed by C Flateau et al. (2010). Investment in supply of effective anti-malarials and anti-retrovirals (ARVs) was significant, and there is also an increasing knowledge about the potential therapeutic implications of the treating co-infected individuals (Vitoria et al, 2009).

Nadjm et al. (2012) in Tanzania stated that severe malaria was diagnosis in $148 / 198$ (74.7\%) cases. $P$. falciparum was identified in $38 / 188(20.2 \%)$ admissions and 26/198 $(13.1 \%)$ were bacteraemic, with the $13 / 25$ (52\%) prescribed empirical antibiotics. HIV was equally common among those with $(16 / 37 ; 43.2 \%)$ and without $P$. falciparum $(50 / 138 ; 36.2 \%)(\mathrm{p}=0.44)$. In $6 / 22(27.3 \%)$ deaths the cause was Streptococcus pneumoniae, Escherichia coli and non-Typhi, Salmonella predominated. Malaria was overdiagnosed in adults with severe febrile illness and was not associated with mortality without co-infection HIV.Mangesho et al. (2014) in Tanzania suggested that drug-drug interactions between certain anti-malarials and anti-retrovials exist affected achieved drug concentrations and may influence the efficacy and/or the safety profiles of those treatments, depending on the medicine combination. They reported that HIV treatment and malaria concurrently often requires individuals to cross domains of different disease programs. In the context of a trial concerned with HIV and malaria, patients experienced the support of clinicians in guiding and reassuring them about when and how to take drugs concomitantly. This presented an 'integrated care' scenario that support patient confidence in concomitant medicine taking following biomedical protocols.

\section{Conclusion}

Malarial anemia is capable of causing severe morbidity and mortality, especially in children and pregnant women. Five species of the malarial parasite infect man. The severity of hematologic disease is related to the ability of the parasites to invade and grow in different red cell populations as well as the intrinsic growth rate of the parasite.

Compared to the non-pregnant women/pregnant/postpartum women are at increased risk of both acquiring malaria and developing more severe disease. One of the unique features of malaria in pregnancy is the ability of 
$P$. falciparum-parasitized erythrocytes to sequester within the intervillous space of the placenta. Placental infection and poor pregnancy outcome decrease in frequency with successive pregnancies for women who reside in endemic areas: nonimmune pregnant women, especially nulliparas, are at higher risk of adverse maternal-perinatal outcome than multiparous women. Pregnancy-associated malaria is characterized by sequestration and multiplication of a distinct population of malarial parasites in the placenta. These parasites express a specific class of variant surface antigens (VSAs) that mediate adhesion of parasite-infected erythrocytes to chondroitin sulfate A (CSA) on the syncytiotrophoblast lining the intervillous space. Once these parasites adhere to the surface of the trophoblastic villi, they induce accumulation of inflammatory leukocytes.

The clinical presentation varies according to the underlying endemicity of the region. Parasitemia peaks in the second trimester in both primigravidas and multigravidas, and the increased risk for pregnancy-associated malaria persists for two months postpartum. Anemia and hypoglycemia are common maternal clinical manifestations.

The diagnosis of malaria should be considered in any febrile pregnant woman who has traveled to or resided in a malarious region, even if briefly or only in transit. Peripheral blood smears are typically used for diagnosis, but may be negative in women with placental malaria who are otherwise asymptomatic. Adverse perinatal outcomes associated with malaria include miscarriage, fetal growth restriction/small for the gestational age infant, preterm birth, low birth weight, perinatal death, and the congenital infected ones.

The imported malaria needs continuous monitoring as many clinicians may not be aware of the zoonotic P. knowlesi in Malaysia extended to the European Union and the America. The travellers back from endemic areas must be laboratory examined. The nosocomial, blood transfusion or even needle-stick transmission is documented.

\section{References}

Aidoo, M, McElroy, PD, Kolczak, MS, et al, 2001: Tumor necrosis factor-alpha promoter variant 2 (TNF2) is associated with pre-term delivery, infant mortality, and malaria morbidity in western Kenya: Asembo Bay Cohort Project IX. Genet. Epidemiol. 21:201-8.

Alweis, RL, DiRosario, K, Conidi, G, Kain, K, Olans, R, et al, 2004: Serial nosocomial transmission of $P$. falciparum malaria from-patient to nurse to patient. Epidemiol. Infect. Control Hosp. 25, 1:55-60.

Angell, SY, Cetron, MS, 2005: Health disparities among travelers visiting friends and relatives abroad. Ann. Int. Med. 142:67-71

Anya, SE, 2004: Seasonal variation in the risk and causes of maternal death in the Gambia: malaria appears to be an important factor. Am. J. Trop. Med. Hyg. 70:510-8.

Arévalo-Herrera, M, Lopez-Perez, M, Medina, L, Moreno, A, Gutierrez, JB, et al, 2015: Clinical profile of Plasmodium falciparum and Plasmodium vivax infections in low and unstable malaria transmission settings of Colombia. Malar. J. 14:154-8.

Askling, HH, Nilsson, J, Tegnell, A, et al, 2005: Malaria risk in travelers. Emerg. Infect. Dis. 11:436-9.

Baird, JK, Fryauff, DJ, Hoffman, SL. 2003: Primaquine for prevention of malaria in travelers. Clin. Infect. Dis. 37:1659-63.

Baird, JK, Sustriayu Nalim, MF, Basri, H, et al, 1996: Survey of resistance to chloroquine by Plasmodium vivax in Indonesia. Trans. R. Soc. Trop. Med. Hyg. 90:409-16.

Bedu-Addo, G, Bates, I, 2002: Causes of massive tropical splenomegaly in Ghana. Lancet 360:449.

Berkley, J, Mwarumba, S, Bramham K, et al, 1999: Bacteraemia complicating severe malaria in children. Trans. R. Soc. Trop. Med. Hyg. 93: 283-9.

Börsch, G, Odendahl, J, Sabin, G, Ricken, D, 1982: Malaria transmission from patient to nurse. Lancet 12:1212-8.

Boele van Hensbroek, M, Calis, JC, Phiri. KS, et al, 2010: Pathophysiological mechanisms of severe anemia in Malawian children. PLoS One 5:e12589. 
Boggild, AK, Parise, ME, Lewis, LS, Kain, K C, 2007: Atovaquone-proguanil: Report from CDC expert meeting on malaria chemoprophylaxis (II). Am. J. Trop. Med. Hyg. 76:208-12.

Bouyou-Akotet, MK, Ionete-Collard, DE, Mabika-Manfoumbi, M, et al, 2003: Prevalence of Plasmodium falciparum infection in pregnant women in Gabon. Malar J. 2:18.

Brabin, BJ, Ginny, M, Sapau, J, et al, 1990: Consequences of maternal anaemia on outcome of pregnancy in a malaria endemic area in Papua New Guinea. Ann. Trop. Med Parasitol. 84:11-9. Bruneel, F, Gachot, B, Wolff, M, et al, 2001: Resurgence of blackwater fever in long-term European expatriates in Africa: report of 21 cases and review. Clin. Infect. Dis. 32:1133-8.

Bruneel, F, Hocqueloux, L, Alberti, C, et al, 2003: The clinical spec-trum of severe imported falciparum malaria in the intensive care unit: report of 188 cases in adults. Am. J. Respir. Crit. Care Med. 167:684-9.

Calis, JC, Phiri, KS, Faragher, EB, et al, 2008: Severe anemia in Malawian children. N. Engl. J. Med. 358:888-94.

Camus, D, Djossou, F, Schilthuis, HJ, et al, 2004: Atovaquone-proguanil versus chloroquine-proguanil for malaria prophylaxis in non-immune pediatric travelers: results of an international, randomized, open-label study. Clin. Infect. Dis. 38, 12:1716-23

Cannon, N, Walker, S, Dismukes, W, 1972: Malaria acquired by accidental needle puncture. JAMA 222:1425-9.

CDC, 2005: Congenital malaria: Nassau County, New York,. Morb Mortal Wkly Rep. 54:383.

CDC, 2008b: Workbook for Designing, Implementing, and Evaluating a Sharps Injury Prevention Program. http://www.cdc.gov/sharpssafeyy/ pdf /sharpswork book.

Chen, JC, Silverthorne, C, 2008: The impact of locus of control on job stress, job performance and job satisfaction in Taiwan. Leadership Organ. Develop. J. 29, 572-82

Chin, W, Contacos, Pg, Coatney, Gr, Kimball, Hr. A, 1965: Naturally acquired quotidiantype malaria in man transferable to monkeys. Sci. 149:865.

Ciminera, P, Brundage, J, 2007: Malaria in U.S. military forces: A description of deployment exposures from 2003 through 2005. Am. J. Trop. Med. Hyg. 76:275-9.

Cornet, M, Le Hesran, JY, Fievet, N, et al, 1998: Prevalence of and risk factors for anemia in young children in southern Cameroon. Am. J. Trop. Med. Hyg. 58:606.

Cox-Singh, J, Davis, TM, Lee, KS, et al, 2008: Plasmodium knowlesi malaria in humans is widely distributed and potentially life threatening. Clin. Infect. Dis. 46:165.

Cox-Singh, J, Davis, TM, Lee, KS, et al, 2008: Plasmodium knowlesi malaria in humans is widely distributed and potentially life threatening. Clin. Infect. Dis. 46:165-9.

Desai, M, ter-Kuile, FO, Nosten, F, et al, 2007: Epidemiology and burden of malaria in pregnancy. Lancet Infect. Dis. 7:93-7.

Dicko, A, Mantel, C, Thera, MA, et al, 2003: Risk factors for malaria infection and anemia for pregnant women in the Sahel area of Bandiagara, Mali. Acta Trop. 89:17-20.

Dorman, EK, Shulman, CE, Kingdom, J, et al, 2002: Impaired uteroplacental blood flow in pregnancies complicated by falciparum malaria. Ultrasound Obstet. Gynecol. 19:165-72.

El-Bahnasawy, MM, Dabbous, HKh, Morsy, TA, 2010: Imported malaria as a threat to Egypt. J. Egypt. Soc. Parasitol., 40, 3:773-87.

Ellis, RD, Fay, MP, Sagara, I, et al, 2011: Anemia in a phase 2 study of a blood stage falciparum malaria vaccine. Malar. J. 10:13-8.

English, M, 2000: Life-threatening severe malarial anaemia. Trans. R. Soc. Trop. Med. Hyg. 94: 585-92.

Flateau, C, Le Loup, G, Pialoux, G, 2011: Consequences of HIV infection on malaria and therapeutic implications: a systematic review. Lancet Infect Dis. 11:541-56.

Fradin, MS, 1998: Mosquitoes and mosquito repellents: A clinician's guide. Ann. Int. Med. 128:931-9.

Fradin, MS, Day, JF, 2002: Comparative efficacy of insect repellents against mosquito bites. N. Engl. J. Med. 347:13-8.

Frances, SP, Waterson, DG, Beebe, NW, Cooper, RD, 2004: Field evaluation of repellent formulations containing DEET and picaridin against mosquitoes in Northern Territory, Australia. J. Med. Entomol. 41:414-8.

Freedman, AM, 1987: Unusual forms of malaria transmission: A report of 2 cases. S. Afr. Med. J. 71:183-4.

Freedman, DO, 2008: Clinical practice: Malaria prevention in short-term travelers. N. Engl. J. Med. 359:603-6.

Fuller, DO, Parenti, MS, Hassan, AN, Beier, JC, 2012: Linking land cover and species distri- 
bution models to project potential ranges of malaria vectors: an example using Anopheles arabiensis in Sudan and Upper Egypt. Malar. J. 11: 264-72.

Gad, AM, Kamel, OM, Abdel Hafez, M, Taha, AM, 1964: A survey of malaria in Sinai, The J. Egypt. Pub. Hlth. Assoc. 39:163-74.

Galappaththy, GN, Omari, AA, Tharyan, P, 2007: Primaquine for preventing relapses in people with Plasmodium vivax malaria. Cochrane Database Syst. Rev.:CD004389.

Greenwood, BM, 1997: The epidemiology of malaria. Ann. Trop. Med. Parasitol.91:763-71. Guerra, CA, Snow, RW, Hay, SI, 2006: Mapping the global extent of malaria in 2005. Trends Parasitol. 22:353-60.

Guerrero, IC, Weniger, BC, Schultz, MG, 1983: Transfusion malaria in the United States, 1972-1981. Ann. Int. Med. 99:221-9.

Hay, SI, Guerra, CA, Tatem, AJ, et al, 2004: The global distribution and population at risk of malaria: past, present, and future. Lancet Infect. Dis. 4:327-32.

Herwaldt, BL, Juranek, DD, 1993: Laboratory-acquired malaria, leishmaniasis, trypanosomiasis, and toxoplasmosis. Am. J. Trop. Med. Hyg. 48, 3:313-23.

Hill, AV, Elvin, J, Willis, AC, et al, 1992: Molecular analysis of the association of HLA-B53 and resistance to severe malaria. Nature 360: 434-6.

Hill, DR, Baird, JK, Parise, ME, et al, 2006: Primaquine: report from CDC expert meeting on malaria chemoprophylaxis I. Am. J. Trop. Med. Hyg. 75:402-8.

Ho, M, Webster, HK, 1989: Immunology of human malaria: a cellular perspective. Parasite Immunol. 11:10516.

Janson, A, 2007: Shed some light on darkness: will Tanzania reach the millennium development goals? Acta Paediatr. 96:781-6.

Kalilani, L, Mofolo, I, Chaponda, M, et al, 2010: The effect of timing and frequency of Plasmodium falciparum infection during pregnancy on the risk of low birth weight and maternal anemia. Trans. R. Soc. Trop. Med. Hyg. 104: 416-20.

Kantele, A, Jokiranta, TS, 2011: Review of cases with the emerging fifth human malaria parasite, Plasmodium knowlesi. Clin. Infect. Dis. 52, 11:1356-62.

Keystone, JS, 2004: The sound of hoof beats does not always mean that it is a zebra. Clin. Inf- ect. Dis. 39:1589-94.

Khan, WA, Galagan, SR, Prue, CS, Khyang, J, Ahmed, S, et al, 2014: Asymptomatic Plasmodium falciparum malaria in pregnant women in the Chittagong Hill Districts of Bangladesh. PLoS One. 9, 5:e98442.

Kimani, EW, Vulule, JM, Kuria, IW, Mugisha, F, 2006: Use of insecticide-treated clothes for personal protection against malaria: a community trial. Malar. J. 5:63-9.

Kochar, DK, Saxena, V, Singh, N, et al, 2005: Plasmodium vivax malaria. Emerg. Infect. Dis. 11:132-8.

Kotton, CN, Ryan, ET, Fishman, JA, 2005: Prevention of infection in adult travelers after solid organ transplantation. Am. J. Transplant. 5: 8-14.

Kublin, JG, Patnaik, P, Jere. CS, et al, 2005: Effect of Plasmodium falciparum malaria on concentration of HIV-1-RNA in the blood of adults in rural Malawi: a prospective cohort study. Lancet 365:233.

Lamikanra, AA, Brown, D, Potocnik, A, et al, 2007: Malarial anemia: of mice and men. Blood 110:18-22.

Lindsay, S, Ansell, JA, Selman, C, et al, 2000: Effect of pregnancy on exposure to malaria mosquitoes [letter]. Lancet 355:1972.

Looareesuwan S, Phillips RE, White NJ, et al, 1985: Quinine and severe falciparum malaria in late pregnancy. Lancet 2:4-5.

Luchavez, J, Espino, F, Curameng, P, et al, 2008: Human Infections with $P$. knowlesi, the Philippines. Emerg. Infect. Dis. 14: 811-8.

Mangesho, PE, Reynolds, J, Lemnge, M, Vestergaard, LS, Chandler, CIR, 2014: Every drug goes to treat its own disease: A qualitative study of perceptions and experiences of taking anti-retrovirals concomitantly with anti-malarials among those affected by HIV and malaria in Tanzania. Malar. J. 13: 491-502.

Maganda, BA, Minzi, OM, Kamuhabwa, AA, Ngasala, B, Sasi, PG, 2014: Outcome of artemether-lumefantrine treatment for uncomplicated malaria in HIV-infected adult patients on anti-retroviral therapy. Malar. J. 13:209-14.

Mali, S, Steele, S, Slutsker, L, Arguin, PM, 2008: Malaria Surveillance-United States. MM WR Surveill. Summ. 57:24-9.

McGready, R, Lee, SJ, Wiladphaingern, J, et al, 2012: Adverse effects of falciparum and vivax malaria and the safety of antimalarial treatment in early pregnancy: A population-based 
study. Lancet Infect Dis 12:388.

McKeage, K, Scott, L, 2003: Atovaquone/ proguanil: a review of its use for the prophylaxis of $P$. falciparum malaria. Drugs 63: 597-608.

Metenou, S, Suguitan, AL,Jr, Long, C, et al, 2007: Fetal immune responses to Plasmodium falciparum antigens in a malaria-endemic region of Cameroon. J Immunol.178:2770.

Modiano, D, Petrarca, V, Sirima, BS, et al, 1996: Different response to Plasmodium falciparum malaria in west African sympatric ethnic groups. Proc. Natl. Acad. Sci. USA 93:13206.

Morris, TJ, Davis, TP, 2000: Doxycyclineinduced esophageal ulceration in the U.S. Military service. Mil. Med.165:316-9.

Morrow, RH, Colebunders, RL, Chin, J, 1989: Interactions of HIV infection with endemic tropical diseases. AIDS 3, 1:S79-87.

Mueller, I, Zimmerman, PA, Reeder, J, 2007: Plasmodium malariae and P. ovale-the "bashful" malaria parasites. Trends Parasitol. 23: 278-82.

Müller, M, Schlagenhauf, P, 2014: Plasmodium knowlesi in travellers, update 2014. Int. J. Infect. Dis. 22:55-64.

Mungai, M, Tegtmeier, G, Chamberland, M, Parise, M, 2001: Transfusion-transmitted malaria in the United States from 1963 through 1999. N. Engl. J. Med. 344:1973-82.

Nadjm, B, Mtove, G, Amos, B, Walker, NF, Diefendal, H, et al, 2012: Severe febrile illness in adult hospital admissions in Tanzania: a prospective study in an area of high malaria transmission. Trans. R. Soc. Trop. Med. Hyg. 106, 11:688-95.

Nahlen, BL, Lobel, HO, Cannon, SE, Campbell, CC, 1991: Reassessment of blood donor selection criteria for United States travelers to malarious areas. Transfusion 31:798-801.

Naqvi, R, Ahmad, E, Akhtar, F, et al, 1996: Predictors of outcome in malarial renal failure. Ren. Fail. 18:685-9.

Nevill, CG, Some, E, Mung'ala, V, et al, 1996: Insecticide-treated bed-nets reduce mortality and morbidity from malaria among children on the Kenyan coast. Trop. Med. Int. Hlth. 1:139-42.

Nosten, F, Kuile, F, Maelankiri, L, et al, 1994: Mefloquine prophylaxis prevents malaria during pregnancy: a double-blind, placebo-controlled study. J. Infect. Dis. 169:595-9.

Okeyeh, JN, Lege-Oguntoye, L, Emembolu, J O, Agbo, M, 1996: Malaria in pregnancy: Efficacy of a low dose of mefloquine in an area holoendemic for multi-drug resistant Plasmodium fa-lciparum. Ann. Trop. Med. Parasitol. 90: 26572.

Orth, H, Jensen, BO, Holtfreter, MC, Kocheril, SJ, Mallach, S, et al, 2013: Plasmodium knowlesi infection imported to Germany, January 2013. Euro. Surveill. Oct 3;18(40). pii: 20603.

Panichakul, T, Payuhakrit, W, Panburana, P, et al, 2012: Suppression of erythroid development in vitro by Plasmodium vivax. Malar. J. 11:173-8.

Piro, S, Sammud, M, Badi, S, Ssabi, L, 2001: Hospital-acquired malaria transmitted by contaminated gloves. J. Hosp. Infect. 47, 2:156-8

Prentice, AM, Doherty, CP, Abrams, SA, et al, 2012: Hepcidin is the major predictor of erythrocyte iron incorporation in anemic African children. Blood 119:1922-30.

Putaporntip, C, Hongsrimuang, T, Seethamchai, S, et al, 2009: Differential prevalence of Plasmodium infections and cryptic Plasmodium knowlesi malaria in humans in Thailand. J. Infect. Dis. 199:1143-8.

Ramharter, M, Grobusch, MP, Kiessling, G, et al, 2005: Clinical and parasitological characteristics of puerperal malaria. J. Infect. Dis. 191: 1005-12.

Ramharter, M, Grobusch, MP, Kiessling, G, et al, 2005: Clinical and parasitological characteristics of puerperal malaria. J. Infect. Dis. 191: 1005-12.

Roberts DJ, Casals-Pascual C, Weatherall DJ, 2005: The clinical and pathophysiological features of malarial anaemia. Curr. Top. Microbiol. Immunol. 295:137-42.

Rodríguez-Morales, AJ, Sánchez, E, Vargas, M, et al, 2006: Anemia and thrombocytopenia in children with Plasmodium vivax malaria. J. Trop. Pediatr. 52:49-54.

Salako, LA, 1984: Toxicity and side-effects of antimalarials in Africa: A critical review. Bull WHO; 62:S63-70.

Salanti A, Dahlbäck M, Turner L, et al, 2004: Evidence for the involvement of VAR2CSA in pregnancy-associated malaria. J. Exp. Med. 200:1197-204.

Saleh, AMA, Adam, SM, Ibrahim, AMA, Morsy, TA, 2016: Malaria: A general minireview with reference to Egypt J. Egypt. Soc. Parasitol. 46, 1:35-48

Saleh, AMA, Adam, SM, Ibrahim, AMA, Morsy, TA, 2017: A training program for nursing staff regarding blood parasites acquired by nee- 
dle-stick injury in a military hospital. J. Egypt. Soc. Parasitol. 47, 1:65-80.

Schellenberg, D, Menendez C, Kahigwa E, et al, 2001: Intermittent treatment for malaria and anaemia control at time of routine vaccinations in Tanzanian infants: a randomised, placebocontrolled trial. Lancet 357:1471.

Schellenberg, D, Schellenberg, JR, Mushi, A, et al, 2003: The silent burden of anaemia in Tanzanian children: a community-based study. Bull. WHO, 81:581.

Schwartz, E, Parise, M, Kozarsky, P, Cetron, M, 2003: Delayed onset of malaria-implications for chemoprophylaxis in travelers. N. Engl. J. Med. 349:1510.

Scott, JA, Berkley, JA, Mwangi, I, et al, 2011: Relation between falciparum malaria and bacteraemia in Kenyan children: a population-based, case-control study and a longitudinal study. Lancet 2011; 378:1316.

Shoukry, NM, Morsy, TA, 2011: Arthropod borne diseases at Toshka, Upper Egypt. World J. Zool. 6, 2:126-33.

Sinka, ME, Bangs, MJ, Manguin, S, Coetzee, M, Mbogo, CM, et al, 2010: The dominant Anopheles vectors of human malaria in Africa, Europe and the Middle East: Occurrence data, distribution maps and bionomic précis, Parasites \&Vectors,3:117,pp.34http:// dx.doi.org/10.1186/ Smoak, BL, Writer, JV, Keep, L, et al, 1997: The effects of inadvertent exposure of mefloquine chemoprophylaxis on pregnancy outcomes and infants of US Army servicewomen. J. Infect. Dis. 176:831-8.

Soto, J, Toledo, J, Rodriquez, M, et al, 1998: Primaquine prophylaxis against malaria in nonimmune Colombian Soldiers: Efficacy and toxicity. Ann. Int. Med. 129:241-5.

Soulard, V, Amadoudji Zin, M, Fitting, C, Ibitokou, S, Oesterholt, M, et al, 2011: Placental malaria-associated suppression of parasitespecific immune response in neonates has no major impact on systemic CD4 T cell homeostasis. Infect. Immun. 79, 7:2801-9.

Strickland, GT, 2000: Hunter's Tropical Medicine and Emerging Infectious Diseases. $8^{\text {th }}$ edition. W.B. Saunders Company..

Subramanian, D, Moise, KJ, Jr, White, AC, Jr, 1992: Imported malaria in pregnancy: report of four cases and review of management. Clin Infect Dis 15:408-12.

Suguitan, AL, Jr, Leke, RG, Fouda, G, et al, 2003: Changes in the levels of chemokines and cytokines in the placentas of women with Plasmodium falciparum malaria. J. Infect. Dis. 188: 1074-80.

Svenson, JE, MacLean, J, Gyorkos, TW, et al, 1995: Imported malaria: Clinical presentation and examination of symptomatic travelers. Arch. Int. Med. 155:861-6.

Tamez, PA, Liu, H, Fernandez-Pol, S, et al, 2009: Stage-specific susceptibility of human erythroblasts to Plasmodium falciparum malaria infection. Blood 114:3652.

Tuikue Ndam, NG, Fievet, N, Bertin, G, et al, 2004: Variable adhesion abilities and overlapping antigenic properties in placental Plasmodium falciparum isolates. J Infect Dis 190:2001-.

UN, 2010: Political definition of "Major regions" Archived from the original on 20 April 2010. UN, 2012: The Millenium Development Goals Report. 2012.

Vadivelan, M, Dutta, T, 2014: Recent advances in the management of Plasmodium knowlesi infection. Trop. Parasitol. 4, 1:31-4.

van Geertruyden, JP, Thomas, F, Erhart, A, D'Alessandro, U, 2004: The contribution of malaria in pregnancy to perinatal mortality. Am J Trop Med Hyg 71:35-9.

van Riemsdijk, MM, Sturkenboom, MC, Ditters, JM, et al, 2002: Atovaquone plus chloroguanide versus mefloquine for malaria prophylaxis: a focus on neuropsychiatric adverse events. Clin. Pharmacol. Ther. 72:294-9.

Vanhauwere, B, Maradit, H, Kerr, L, 1998: Post-marketing surveillance of prophylactic mefloquine (Lariam) use in pregnancy. Am. J. Trop. Med. Hyg. 58:17-22.

Vitoria, M, Granich, R, Gilks, CF, Gunneberg, C, Hosseini, M, et al, 2009: The global fight against HIV/AIDS, tuberculosis, and malaria. Am. J. Clin Pathol. 131:844-8.

Wallace, MR, Sharp, T, Smoak, B, et al, 1996: Malaria among United States troops in Somalia. Am. J. Med. 100:49-51.

Wellems, TE, Plowe, CV, 2001: Chloroquineresistant malaria. J. Infect. Dis. 184:770.

WHO, 2010: World Malaria Report. Geneva. WHO, 2012: Global Malaria Program, Geneva. Wilson, J, Loveday, H, Hoffman, P, 2007: Uniform: an evidence review of the microbiological significance of uniforms and uniform policy in the prevention and control of healthcare-associated infections: report to the Department of Health (England). J. Hosp. Infect. 66: 301-7. 